\title{
Hausdorff dimension and Diophantine approximation
}

\author{
Yann Bugeaud
}

\begin{abstract}
In the present survey paper, we explain how the theory of Hausdorff dimension and Hausdorff measure is used to answer certain questions in Diophantine approximation. The final section is devoted to a discussion around the Diophantine properties of the points lying in the middle third Cantor set.
\end{abstract}

\section{Introduction}

The main goal of this survey paper is to point out how the theory of Hausdorff dimension and Hausdorff measure can be used to solve various questions in Diophantine approximation. We also point out several open problems, which hopefully will motivate further research.

Throughout this text we denote by dim the Hausdorff dimension.

Let $\xi$ be an irrational real number. By the theory of continued fractions (or by Dirchlet's Schubfachprinzip), there exist infinitely many rational numbers $p / q$ such that

$$
|\xi-p / q|<q^{-2} \text {. }
$$

For any given $\varepsilon>0$, a covering argument (easy half of the Borel-Cantelli lemma) shows that, for almost all real numbers $\xi$ (throughout Sections 1 and 2, 'almost all' always refers to the Lebesgue measure), there are only finitely many rational numbers $p / q$ such that

$$
|\xi-p / q|<q^{-2-\varepsilon} \text {. }
$$

However, for certain irrational real numbers $\xi$, inequality (1.1) has infinitely many solutions.

Definition 1.1. The irrationality exponent of an irrational real number $\xi$, denoted by $\mu(\xi)$, is the supremum of the real numbers $\mu$ such that

$$
|\xi-p / q|<q^{-\mu}
$$

has infinitely many solutions in rational numbers $p / q$.

2000 Mathematics Subject Classification : 11J04, 11J13, 11J83. 
By means of the theory of continued fractions, for every real number $\mu \geq 2$, it is easy to construct real numbers $\xi$ such that $\mu(\xi)=\mu$; see e.g. [17] or [12]. Moreover, Jarník [16] proved in 1929 that

$$
\operatorname{dim}\{\xi \in[0,1]: \mu(\xi) \geq \mu\}=\frac{2}{\mu},
$$

a result established independently by Besicovitch [8] a few years later. In 1931 Jarník [17] refined his result from [16] by using generalized Hausdorff measures. Although this is not explicitly written in [17], he showed that

$$
\operatorname{dim}\{\xi \in[0,1]: \mu(\xi)=\mu\}=\frac{2}{\mu} .
$$

The irrationality exponent $\mu$ introduced in Definition 1.1 is an example of an exponent of approximation, that is, of a function defined on the set of real irrational numbers by means of consideration from Diophantine approximation.

Definition 1.2. The spectrum of an exponent of approximation is the set of values taken by this exponent.

For an exponent of approximation $w$, there are two natural questions:

* (Q1) To determine the spectrum of $w$;

* (Q2) For a real number $w_{0}$, to determine the Hausdorff dimension of the set at level $w_{0}$, that is, of the set of real numbers $\xi$ such that $w(\xi)=w_{0}$.

In the case of the irrationality exponent, we have seen above that both questions have been answered. We introduce in Section 2 three (classical) families of exponents of approximation and discuss both questions for these exponents. We will see that, in certain cases, to answer (Q2) is the only known way to solve the apparently simpler (Q1).

Section 3 is devoted to a survey of recent results on Diophantine approximation of elements of the middle third Cantor set.

\section{Three families of exponents of approximation}

We define two families of exponents of approximation which generalize the irrationality exponent. A third family of exponents gives certain information on the expansions of real numbers to integer bases. Further classical families of exponents are discussed in [13].

For $n \geq 1$, the accuracy with which real numbers are approximated by algebraic numbers of degree at most $n$ is measured by means of the exponents $w_{n}^{*}$, introduced in 1939 by Koksma [19]. Recall that the height $H(P)$ of an integer polynomial $P(X)$ is the maximum of the moduli of its coefficients, and the height $H(\alpha)$ of an algebraic number $\alpha$ is the height of its minimal polynomial over $\mathbf{Z}$.

Definition 2.1. Let $n \geq 1$ be an integer and let $\xi$ be an irrational real number. We denote by $w_{n}^{*}(\xi)$ the supremum of the real numbers $w^{*}$ for which the inequality

$$
|\xi-\alpha| \leq H(\alpha)^{-w^{*}-1}
$$

is satisfied for infinitely many algebraic numbers $\alpha$ of degree at most $n$. 
Clearly, every irrational real number $\xi$ satisfies

$$
\mu(\xi)=w_{1}^{*}(\xi)+1
$$

This shows that the exponents $w_{n}^{*}$ with $n \geq 2$ extend in a natural way the irrationality exponent $\mu$.

The introduction of the exponent -1 in $(2.1)$ is explained on page 48 of [10]. The reader is directed to this monograph for known results on the exponents $w_{n}^{*}$. We only mention here that $w_{n}^{*}(\xi)=\min \{n, d-1\}$ for every real algebraic number $\xi$ of degree $d \geq 2$ and that almost all real numbers $\xi$ satisfy $w_{n}^{*}(\xi)=n$ for $n \geq 1$. Wirsing [25] proved that $w_{n}^{*}(\xi) \geq(n+1) / 2$ for every transcendental real number $\xi$ and every $n \geq 1$. With the exception of a result of Davenport and Schmidt [14], showing that $w_{2}^{*}(\xi) \geq 2$ for every real number $\xi$ not of degree at most 2, there has been no significant improvement of Wirsing's statement during the last fifty years.

Problem 2.2. Are there an integer $n \geq 3$ and a transcendental real number $\xi$ such that $w_{n}^{*}(\xi)<n ?$

Other natural exponents of approximation, which also extend the irrationality exponent $\mu$, take into account the accuracy with which the first integral powers of a given real number are simultaneously approximated by rational numbers with the same denominator. Throughout this text, $\|$.$\| denotes the distance to the nearest integer.$

Definition 2.3. Let $n \geq 1$ be an integer and let $\xi$ be an irrational real number. We denote by $\lambda_{n}(\xi)$ the supremum of the real numbers $\lambda$ for which the inequality

$$
\max _{1 \leq m \leq n}\left\|q \xi^{m}\right\| \leq q^{-\lambda}
$$

has infinitely many solutions $q$ in $\mathbf{Z}_{\geq 1}$.

We have $\lambda_{n}(\xi)=\max \{1 / n, 1 /(d-1)\}$ for $n \geq 1$ for every real algebraic number $\xi$ of degree $d \geq 2$. Furthermore, $\lambda_{n}(\xi) \geq 1 / n$ for every irrational real number $\xi$ and every $n \geq 1$; see [12] for further results.

We end this list of definitions with the exponents $v_{b}$ which were introduced in [1]. They provide information on the lengths of blocks of digits 0 (or of digits $b-1$ ) occurring in the expansion of $\xi$ to base $b$.

Definition 2.4. Let $\xi$ be an irrational real number. Let $b$ be an integer with $b \geq 2$. We denote by $v_{b}(\xi)$ the supremum of the real numbers $v$ for which the inequality

$$
\left\|b^{n} \xi\right\|<\left(b^{n}\right)^{-v}
$$

has infinitely many solutions $n$ in $\mathbf{Z}_{\geq 1}$.

Let $b \geq 2$ be an integer. Clearly, we have

$$
w_{1}^{*}(\xi) \geq v_{b}(\xi), \quad \mu(\xi) \geq v_{b}(\xi)+1
$$


for all irrational real numbers $\xi$. These inequalities are rarely sharp since almost all real numbers $\xi$ satisfy $v_{b}(\xi)=0$.

For every $b \geq 2$ and for every $v$ with $0<v<+\infty$ the real number

$$
\xi_{b, v}:=\sum_{j \geq 1} b^{-\left\lfloor(v+1)^{j}\right\rfloor},
$$

where $\lfloor$.$\rfloor denotes the integer part, satisfies v_{b}\left(\xi_{b, v}\right)=v$. Since

$$
v_{b}\left(\sum_{j \geq 1} b^{-j^{2}}\right)=0 \quad \text { and } \quad v_{b}\left(\sum_{j \geq 1} b^{-j !}\right)=+\infty
$$

this shows that the spectrum of $v_{b}$ is equal to $[0,+\infty]$. Thus, we have given the answer to (Q1) for the exponent $v_{b}$.

Now, we explain how to show that, for every real number $v>0$, we have

$$
\operatorname{dim}\left\{\xi \in[0,1]: v_{b}(\xi) \geq v\right\}=\operatorname{dim}\left\{\xi \in[0,1]: v_{b}(\xi)=v\right\}=\frac{1}{v+1} .
$$

To bound this Hausdorff dimension from above by $1 /(v+1)$ is an immediate application of a covering argument (easy half of the Hausdorff-Cantelli lemma). To prove that this dimension is at least equal to $1 /(v+1)$ is much more interesting. We construct inductively a large Cantor-type set contained in $\left\{\xi \in[0,1]: v_{b}(\xi) \geq v\right\}$. Let $\left(n_{k}\right)_{k \geq 1}$ be a rapidly increasing sequence of integers with $n_{1}=1$ and $n_{2}>2 / v$. Set $\mathcal{E}_{1}=[0,1]$. For $p=$ $1, \ldots, b^{n_{2}}-1$, set

$$
E_{2, p}=\left[p / b^{n_{2}}-1 / b^{(v+1) n_{2}}, p / b^{n_{2}}+1 / b^{(v+1) n_{2}}\right]
$$

and put $\mathcal{E}_{2}=E_{2,1} \cup \ldots \cup E_{2, b^{n}-1}$. Assume that, for some $k \geq 2$, the set $\mathcal{E}_{k}$ has been constructed and is equal to a finite union of intervals $E_{k, 1}, \ldots, E_{k, t_{k}}$ of length $2 b^{-(v+1) n_{k}}$ and centered at rational numbers of denominator $b^{n_{k}}$. Let $E_{k, p}$ be such an interval. Let denote by $\mathcal{E}_{k+1, p}$ the set of intervals of the form

$$
\left[a / b^{n_{k+1}}-1 / b^{(v+1) n_{k+1}}, a / b^{n_{k+1}}+1 / b^{(v+1) n_{k+1}}\right]
$$

with $a$ an integer, which are contained in $E_{k, p}$. There are at least

$$
m_{k+1}:=b^{n_{k+1}}\left(2 b^{-(v+1) n_{k}}\right)-2
$$

such intervals and the distance between any two distinct such intervals always exceeds

$$
\varepsilon_{k+1}:=b^{-n_{k+1}} / 2
$$

Putting $\mathcal{E}_{k+1}=\mathcal{E}_{k+1,1} \cup \ldots \cup \mathcal{E}_{k+1, t_{k}}$, we have completed the inductive step of the construction. Set

$$
\mathcal{K}:=\bigcap_{k \geq 1} \mathcal{E}_{k}
$$


By construction, every element $\xi$ in $\mathcal{K}$ satisfies $v_{b}(\xi) \geq v$. The mass distribution principle (as stated e.g. on page 59 of [15] or on page 97 of [10]) shows that

$$
\operatorname{dim} \mathcal{K} \geq \liminf _{k \rightarrow+\infty} \frac{\log \left(m_{1} \ldots m_{k}\right)}{-\log \left(m_{k+1} \varepsilon_{k+1}\right)}
$$

In our situation, if the sequence $\left(n_{k}\right)_{k \geq 1}$ grows sufficiently rapidly, we deduce that

$$
\operatorname{dim}\left\{\xi \in[0,1]: v_{b}(\xi) \geq v\right\} \geq \frac{1}{v+1}
$$

To get the same lower bound for the smaller set

$$
\operatorname{dim}\left\{\xi \in[0,1]: v_{b}(\xi)=v\right\}
$$

we need to use refined Hausdorff measures, but there is no additional difficulty.

In the above proof, we have used that, if $I$ is an interval of positive length $|I|$ contained in $[0,1]$, then, for $n$ large enough in terms of $|I|$, there are around $b^{n}|I|$ rational points of the form $a / b^{n}$ in $I$, and these points are regularly spaced.

The same strategy was used by Jarník to establish (1.2) and (1.3). He proved that the set of rational numbers $p / q$ in $[0,1]$ is evenly distributed in the following sense: For $I$ as above, if $Q$ is large enough in terms of $|I|$, then there are $\gg Q^{2}$ rational numbers $p / q$ in $I$ with $Q \ll q \ll Q$ and such that the distance between any two of them is $\gg Q^{-2}$ (here and below, the constants implicit in $\ll$ and $\gg$ are numerical constants). This is proved on page 99 of [10]; see also on page 142 of [15] for a weaker result, however sufficient to derive (1.2) and (1.3).

To formalize the properties of distribution needed to apply the method described above, Baker and Schmidt [2] have introduced the notion of regular systems, see Section 5.4 of [10]. Several authors prefer to use ubiquitous systems [6], which give more flexibility.

For $n \geq 1$, explicit examples of real numbers have been constructed in [12] in order to show that the spectrum of $w_{n}^{*}$ includes the interval $[2 n-1,+\infty)$. However, the next question remains open.

Problem 2.5. Let $n \geq 2$ be an integer. To construct explicitly a real number $w$ with $n<w<2 n-1$ and a real number $\xi$ such that $w_{n}^{*}(\xi)=w$.

Apparently, there is no suitable multidimensional generalisation of the theory of continued fraction which can be used to solve (at least partially) Problem 2.5.

At present, for $n \geq 2$, the only known way to show that the spectrum of $w_{n}^{*}$ includes the interval $[n,+\infty)$ is by means of the next theorem, proved in 1970 by Baker and Schmidt $[2]$.

Theorem 2.6. Let $n \geq 1$ be an integer. For every real number $w^{*} \geq n$, we have

$$
\operatorname{dim}\left\{\xi \in[0,1]: w_{n}^{*}(\xi)=w^{*}\right\}=\frac{n+1}{w^{*}+1} .
$$


We check that (1.3) and (2.5) for $n=1$ coincide. Baker and Schmidt used the same strategy as explained above. The difficult point is to prove that algebraic numbers of bounded degree are evenly distributed. Note that, since an algebraic number of degree $n$ and height $H$ is a root of an integer polynomial of degree $n$ and with all coefficients bounded in absolute value by $H$, their number does not exceed $(2 H+1)^{n+1}$. With $I$ as above, it is proved in [2] that, if $H$ is large enough in terms of $|I|$, then there are $\gg H^{n+1}|I|$ algebraic numbers $\alpha$ of degree $n$ in $I$ with $H(\alpha) \ll H(\log H)^{c(n)}$ and such that the difference between any two of them if $\gg H^{-n-1}$. Here, $c(n)$ is a constant depending only on $n$. A deep result of Beresnevich [4] from 1999 shows that the above statement remains true with $c(n)=0$.

Thus, we have seen that (Q1) and (Q2) are answered for the exponents of approximation $v_{b}$ and $w_{n}^{*}$. The situation is different and much more complicated for the exponents $\lambda_{n}$.

For $n \geq 1$, Bugeaud [12] constructed explicit examples of real numbers in order to show that the spectrum of $\lambda_{n}$ includes the interval $[1,+\infty)$. However, the next question remains open.

Problem 2.7. Let $n \geq 2$ be an integer. To construct explicitly a real number $\lambda$ with $1 / n<\lambda<1$ and a transcendental real number $\xi$ such that $\lambda_{n}(\xi)=\lambda$.

At present, the only known way to show that the spectrum of $\lambda_{2}$ is the whole interval $[1 / 2,+\infty)$ is the proof by Beresnevich, Dickinson, Vaughan and Velani $[7,23]$ that, for every $\lambda$ in $[1 / 2,1]$, we have

$$
\operatorname{dim}\left\{\xi \in[0,1]: \lambda_{2}(\xi)=\lambda\right\}=\frac{2-\lambda}{1+\lambda} .
$$

The Jarník-Besicovitch theorem (1.3) was recently extended by Budarina, Dickinson, and Levesley [9] as follows (see also [12] for an alternative proof).

Theorem 2.8. Let $n \geq 2$ be an integer. Let $\lambda \geq n-1$ be a real number. Then, we have

$$
\operatorname{dim}\left\{\xi \in[0,1]: \lambda_{n}(\xi)=\lambda\right\}=\frac{2}{n(1+\lambda)} .
$$

The assumption $\lambda \geq n-1$ in Theorem 2.8 is quite restrictive when $n \geq 3$ but is optimal for $n=2$.

We end this section with two open questions.

Problem 2.9. Let $n \geq 3$ be an integer. To determine the spectrum of $\lambda_{n}$.

Problem 2.10. Let $n \geq 3$ be an integer. Let $\lambda$ be a real number with $1 / n<\lambda<n-1$. To determine

$$
\operatorname{dim}\left\{\xi \in[0,1]: \lambda_{n}(\xi)=\lambda\right\} .
$$

Partial results towards the resolution of the difficult Problems 2.9 and 2.10 have been given by Beresnevich [5]. He proved that, for an integer $n$ and a real number $\lambda$ satisfying $1 / n \leq \lambda<3 /(2 n-1)$, we have

$$
\operatorname{dim}\left\{\xi \in[0,1]: \lambda_{n}(\xi) \geq \lambda\right\} \geq \frac{n+1}{\lambda+1}-(n-1) .
$$

He conjectured that equality holds in (2.6). 


\section{Approximation to points in the middle third Cantor set}

Throughout this section, we denote by $K$ the middle third Cantor set. Certain results stated below are valid for more general Cantor-type sets, however. We recall that the Hausdorff dimension of $K$ satisfies

$$
\operatorname{dim} K=\frac{\log 2}{\log 3}
$$

The first significant result on Diophantine approximation to elements of $K$ was proved in 2001 by Weiss [24]. He showed that the irrationality exponent of almost all $\xi$ in $K$ (in this section, almost all refers to the standard measure supported by $K$ ) is equal to 2 .

For $v>0$, the real number $\xi_{3, v}$ defined in (2.3) satisfies

$$
v_{3}\left(\xi_{3, v}\right)=v, \text { and thus } \mu\left(\xi_{3, v}\right) \geq v+1
$$

by (2.2) or by noticing that

$$
\left|\xi_{3, v}-\sum_{j=1}^{J} 3^{-\left\lfloor(v+1)^{j}\right\rfloor}\right|<2 \cdot 3^{-\left\lfloor(v+1)^{J+1}\right\rfloor} \leq 6 \cdot\left(3^{-\left\lfloor(v+1)^{J}\right\rfloor}\right)^{v+1},
$$

for every sufficiently large integer $J$. At first sight, for $v \geq 1$, it could seem that $\xi_{3, v}$ satisfies

$$
\mu\left(\xi_{3, v}\right)=v_{3}\left(\xi_{3, v}\right)+1=v+1 .
$$

This is however not clear since there may exist very good rational approximants to $\xi_{3, v}$ which are not obtained by truncation of the infinite sum giving $\xi_{3, v}$, that is, which are not of the form $\sum_{j=1}^{J} 3^{-\left\lfloor(v+1)^{j}\right\rfloor}$ for some $J \geq 1$. However, if $v$ is sufficiently large - precisely, if $v \geq(\sqrt{5}+1) / 2-$, a simple argument based on triangle inequalities (see e.g. Section 8 of [21]) implies (3.1).

In fact, it appears that (3.1) holds for every $v \geq 1$, as proved in [11] by means of the following observation: the continued fraction expansion of a suitable rational translate of $-\xi_{3, v}$ can be constructed explictly. The next result is extracted from [11].

Theorem 3.1. Let $\mu \geq 2$ be a real number. The middle third Cantor set contains uncountably many elements $\xi$ with $\mu(\xi)=\mu$.

Theorem 3.1 answers (Q1) for the restriction to $K$ of the irrationality exponent, but (Q2) remains open. In order to attack the latter question, the authors of [21] investigated the analogous problem for the exponent $v_{3}$. They proved the following statement.

Theorem 3.2. For any positive real number $v$ we have

$$
\begin{aligned}
\operatorname{dim}\left\{\xi \in K: v_{3}(\xi)=v\right\} & =\frac{\log 2}{\log 3} \times \frac{1}{v+1} \\
& =(\operatorname{dim} K) \times \operatorname{dim}\left\{\xi \in[0,1]: v_{3}(\xi)=v\right\}
\end{aligned}
$$


Things are more easier with the exponent $v_{3}$ than with the irrationality exponent $\mu$. Indeed, if for some $v>1$ and $\xi$ in $K$ we have

$$
\left|\xi-p / 3^{n}\right|<\left(3^{n}\right)^{-v}
$$

for some rational number $p / 3^{n}$, then $p / 3^{n}$ must lie in $K$.

We display an immediate consequence of (1.3), (2.4), and Theorem 3.2.

Corollary 3.3. For any real number $\mu>2$, we have

$$
\operatorname{dim}\{\xi \in K: \mu(\xi) \geq \mu\} \geq \frac{1}{2} \times(\operatorname{dim} K) \times \operatorname{dim}\{\xi \in[0,1]: \mu(\xi)=\mu\} .
$$

Apparently, the methods used in [21] do not allow us to replace the $\geq$ sign by the $=$ sign in the left hand side of (3.2). They, however, give the following slight refinement of $(3.2)$ :

$$
\operatorname{dim}\{\xi \in K: \mu \leq \mu(\xi) \leq 2 \mu\} \geq \frac{1}{2} \times(\operatorname{dim} K) \times \operatorname{dim}\{\xi \in[0,1]: \mu(\xi)=\mu\} .
$$

Regarding the upper bound, Pollington and Velani [22] (see also [20]) used a simple covering argument to establish that

$$
\operatorname{dim}\{\xi \in K: \mu(\xi) \geq \mu\} \leq(\operatorname{dim} K) \times \operatorname{dim}\{\xi \in[0,1]: \mu(\xi) \geq \mu\},
$$

for every $\mu \geq 2$.

Let $\mu>2$ be a real number. The authors of [21] speculate at the end of their paper that we have

$$
\operatorname{dim}\{\xi \in K: \mu(\xi) \geq \mu\}=(\operatorname{dim} K) \times \operatorname{dim}\{\xi \in[0,1]: \mu(\xi) \geq \mu\} .
$$

Problem 3.4. Let $\mu>2$ be a real number. To determine

$$
\operatorname{dim}\{\xi \in K: \mu(\xi)=\mu\} .
$$

A related question involving rational approximation and asymptotic frequencies of digits in a fixed integer base has been investigated in [3].

Very few is known on the expansions of a given irrational real number to two multiplicatively independent bases. The study of the set of values taken by the exponent $v_{2}$ at the points in $K$ would give some information on the binary expansions of the elements of $K$.

A straightforward adaptation of the arguments of Weiss [24] and Kristensen [20] allows us to prove the next result.

Theorem 3.5. For any positive real number $v$ we have

$$
\operatorname{dim}\left\{\xi \in K: v_{2}(\xi) \geq v\right\} \leq(\operatorname{dim} K) \times \operatorname{dim}\left\{\xi \in[0,1]: v_{2}(\xi) \geq v\right\} .
$$

However, it does not seem to be easy to give a non-trivial lower bound for the left-hand side of (3.3). 
Problem 3.6. Let $v$ be a positive real number. To determine

$$
\operatorname{dim}\left\{\xi \in K: v_{2}(\xi)=v\right\} .
$$

Weiss' result mentioned at the beginning of this section has been extended in Proposition 7.10 from [18] to the exponents $w_{n}^{*}$.

Theorem 3.7. Almost all points $\xi$ in the middle third Cantor set satisfy

$$
w_{n}^{*}(\xi)=n, \quad \text { for every } n \geq 1
$$

and

$$
\lambda_{n}(\xi)=1 / n, \quad \text { for every } n \geq 1 .
$$

The last part of Theorem 3.7 follows by combining Proposition 7.10 from [18] with a classical transference principle. We omit the details.

We conclude this text by a last open problem; see Section 6 of [12] for a small contribution towards its resolution.

Problem 3.8. Let $n \geq 1$ be an integer, $w \geq n$ and $\lambda \geq 1 / n$ be real numbers. To determine the Hausdorff dimension of the sets

$$
\left\{\xi \in K: w_{n}^{*}(\xi)=w\right\}
$$

and

$$
\left\{\xi \in K: \lambda_{n}(\xi)=\lambda\right\} .
$$

For $n \geq 2$, to determine the spectra of the restriction to $K$ of $w_{n}^{*}$ and of $\lambda_{n}$.

\section{References}

[1] M. Amou and Y. Bugeaud, Expansions in integer bases and exponents of Diophantine approximation, J. London Math. Soc. 81 (2010), 297-316.

[2] A. Baker and W. M. Schmidt, Diophantine approximation and Hausdorff dimension, Proc. London Math. Soc. 21 (1970), 1-11.

[3] J. Barral and S. Seuret, Ubiquity and large intersections properties under digit frequencies constraints, Math. Proc. Cambridge Philos. Soc. 145 (2008), 527-548.

[4] V. Beresnevich, On approximation of real numbers by real algebraic numbers, Acta Arith. 90 (1999), 97-112.

[5] V. Beresnevich, Rational points near manifolds and metric Diophantine approximation, Ann. of Math. 175 (2012), 187-235.

[6] V. Beresnevich, D. Dickinson and S. L. Velani, Measure Theoretic Laws for limsup Sets, Mem. Amer. Math. Soc. 179 (2006), no. 846, 1-91. 
[7] V. Beresnevich, D. Dickinson and S. L. Velani, Diophantine approximation on planar curves and the distribution of rational points, with an appendix by R.C. Vaughan: 'Sums of two squares near perfect squares', Ann. of Math. 166 (2007), 367-426.

[8] A. S. Besicovitch, Sets of fractional dimension (IV): on rational approximation to real numbers, J. London Math. Soc. 9 (1934), 126-131.

[9] N. Budarina, D. Dickinson, and J. Levesley, Simultaneous Diophantine approximation on polynomial curves. Mathematika 56 (2010), 77-85.

[10] Y. Bugeaud, Approximation by algebraic numbers, Cambridge Tracts in Mathematics, Cambridge, 2004.

[11] Y. Bugeaud, Diophantine approximation and Cantor sets, Math. Ann. 341 (2008), $677-684$.

[12] Y. Bugeaud, On simultaneous rational approximation to a real number and its integral powers, Ann. Inst. Fourier (Grenoble) 60 (2010), 2165-2182.

[13] Y. Bugeaud and M. Laurent, Exponents of Diophantine approximation. In: Diophantine Geometry Proceedings, Scuola Normale Superiore Pisa, Ser. CRM, vol. 4, 2007, 101-121.

[14] H. Davenport and W. M. Schmidt, Approximation to real numbers by quadratic irrationals, Acta Arith. 13 (1967), 169-176.

[15] K. Falconer, Fractal Geometry : Mathematical Foundations and Applications, John Wiley \& Sons, 1990.

[16] V. Jarník, Diophantischen Approximationen und Hausdorffsches Mass, Mat. Sbornik 36 (1929), 371-382.

[17] V. Jarník, Über die simultanen Diophantische Approximationen, Math. Z. 33 (1931), $505-543$.

[18] D. Kleinbock, E. Lindenstrauss, and B. Weiss, On fractal measures and Diophantine approximation, Selecta Math. 10 (2004), 479-523.

[19] J. F. Koksma, Über die Mahlersche Klasseneinteilung der transzendenten Zahlen und die Approximation komplexer Zahlen durch algebraische Zahlen, Monatsh. Math. Phys. 48 (1939), 176-189.

[20] S. Kristensen, Approximating numbers with missing digits by algebraic numbers, Proc. Edinb. Math. Soc. 49 (2006), 657-666.

[21] J. Levesley, C. Salp and S. L. Velani, On a problem of K. Mahler: Diophantine approximation and Cantor sets, Math. Ann. 338 (2007), 97-118.

[22] A. D. Pollington and S. Velani, Metric Diophantine approximation and 'absolutely friendly' measures, Selecta Math. 11 (2005), 297-307.

[23] R. C. Vaughan and S. Velani, Diophantine approximation on planar curves: the convergence theory, Invent. Math. 166 (2006), 103-124. 
[24] B. Weiss, Almost no points on a Cantor set are very well approximable, R. Soc. Lond. Proc. Ser. A Math. Phys. Eng. Sci. 457 (2001), 949-952.

[25] E. Wirsing, Approximation mit algebraischen Zahlen beschränkten Grades, J. reine angew. Math. 206 (1961), 67-77.

Yann Bugeaud

Université de Strasbourg

Mathématiques

7, rue René Descartes

67084 STRASBOURG (FRANCE)

bugeaud@math.unistra.fr 